\title{
Using prolonged breakthroughs of a nitrate rich agricultural catchment to gain insight into its configuration and hydro-chemical processes therein via modelling the nitrate travel times.
}

\author{
BAIBASWATA BHADURI ${ }^{1}$, SEKHAR MUDDU ${ }^{1}$ AND \\ LAURENT RUIZ ${ }^{2}$ \\ ${ }^{1}$ Indian Institute of Science \\ ${ }^{2}$ Indian Institute of Science, Bangalore \\ Presenting Author: baibaswatabhaduri@gmail.com
}

Intensification of agriculture and consequent anthropogenic inputs of nitrogen in agricultural watersheds of certain European provinces over the last few decades has significantly increased the ground water concentration of nitrates, which isn't being expelled expeditiously despite strict regulations. Le Puits, one such agricultural nested headwater catchment in French Brittany, being extensively monitored by AgrHys Critical Zone Observatory for an extended period, became a hotspot for environmental modelers and hydrogeologists interested in process based integrative modelling approach for understanding of the key processes in such complex subsurface structures by observing the Travel Time of Nitrates. We thus designed a semidistributed semi-analytical reactor-network based model using concepts of chemical reaction engineering and control theory to determine the Transit Time Distribution of groundwater systems, and applied it to Le Puits to gain insight into the relative contribution of different nested subcatchments in reproducing the major breakthrough at the outlet. Results reveal that the reason behind the attenuated response of the legacy contamination can principally be attributed to compartmentalization effects - the presence of deep, interactive groundwater chambers delays the flushing time of Nitrates in such a way that the applied managerial measures take several years to display any footprint downstream. Defiance of universal fractal scaling (Kirchner and Neal, 2013) of the breakthroughs by exhibition of very steep power-spectra slopes (brown-noise) further established the memory effects. More interestingly, despite Le Puits' small size, there are stark differences between the Nitrate flushing times within it's different subcatchments - they selectively contribute in reproducing either the seasonality or the prolonged tails of the Nitrate Breakthrough at the main outlet, emphasizing the structural dissimilarity and variegated solute dispersivity amongst different parts of the catchment of same geogenic origin. Our model, owing to its semi-distributed nature, being also applicable to catchments monitored via piezometers only, in future we plan to apply the same to Kabini Critical Zone Observatory in India, where the river is largely dry.

\section{References:}

- Kirchner JW, Neal C. 2013. Universal fractal scaling in stream chemistry and its implications for solute transport and water quality trend detection. Proceedings of the National Academy of Sciences 110(30): 1221312218 . 